\title{
Qualitative Analysis of Gradient-Type Systems with Oscillatory Nonlinearities on the Sierpiński Gasket*
}

\author{
Gabriele BONANNO ${ }^{1}$ Giovanni MOLICA BISCI ${ }^{2}$ Vicenţiu RĂDULESCU ${ }^{3}$
}

\begin{abstract}
Under an appropriate oscillating behavior either at zero or at infinity of the nonlinear data, the existence of a sequence of weak solutions for parametric quasilinear systems of the gradient-type on the Sierpiński gasket is proved. Moreover, by adopting the same hypotheses on the potential and in presence of suitable small perturbations, the same conclusion is achieved. The approach is based on variational methods and on certain analytic and geometrical properties of the Sierpiński fractal as, for instance, a compact embedding result due to Fukushima and Shima.
\end{abstract}

Keywords Sierpiński gasket, Nonlinear elliptic equation, Dirichlet form, Weak Laplacian

2000 MR Subject Classification 35J20, 28A80, 35J25, 35J60, 47J30, 49J52

\section{Introduction}

A particular interest has been given in the last few decays to the study of various nonlinear partial differential equations on fractal domains. For instance, many physical problems lead to nonlinear models involving reaction-diffusion equations, problems on elastic fractal media or fluid flow through fractal regions. We also point out relevant applications of the fractal theory to topology, differential geometry, functional and harmonic analysis, and probability theory. For recent advances in the theory of nonlinear elliptic equations on fractals we refer to Barlow and Kigami [2], Bockelman and Strichartz [6], Falconer [24], Falconer and Hu [26], Hu [30], Hua and Zhenya [31], and Strichartz [44-45]. The main tools used in some of these papers to prove the existence of nontrivial solutions or multiple solutions to nonlinear elliptic equations with zero Dirichlet boundary conditions defined on fractals are certain minimax results (mountain pass or saddle point type theorems), results from genus theory, and minimization procedures. A particular concern has been devoted to PDEs on the Sierpiński gasket.

\footnotetext{
Manuscript received October 26, 2012.

${ }^{1}$ Department of Science for Engineering and Architecture (Mathematics Section) Engineering Faculty,

University of Messina, 98166 Messina, Italy. E-mail: bonanno@unime.it

${ }^{2}$ Department Patrimonio Architettonico e Urbanistico PAU Università degli Studi "Mediterranea" di Reggio Calabria Via Melissari, I-89124 Reggio Calabria, Italy. E-mail: gmolica@unirc.it

${ }^{3}$ Institute of Mathematics "Simion Stoilow" of the Romanian Academy, P. O. Box 1-764, 014700 Bucharest, Romania; Department of Mathematics, University of Craiova, Street A. I. Cuza No. 13, 200585 Craiova, Romania. E-mail: vicentiu.radulescu@imar.ro

*Vicenţiu Rădulescu was supported by Grant CNCS PCE 47/2011 (Qualitative and Numerical Analysis of Nonlinear Problems on Fractals). The second author was supported by the GNAMPA Project (Esistenza e molteplicitá di soluzioni per problemi differenziali non lineari) 2012.
} 
In the present paper, we are interested in Dirichlet gradient-type systems of the form

$$
\begin{cases}\Delta u_{1}(x)+a_{1}(x) u_{1}(x)=\lambda g(x) F_{u_{1}}\left(u_{1}(x), u_{2}(x)\right), & x \in V \backslash V_{0}, \\ \Delta u_{2}(x)+a_{2}(x) u_{2}(x)=\lambda g(x) F_{u_{2}}\left(u_{1}(x), u_{2}(x)\right), & x \in V \backslash V_{0}, \\ \left.u_{1}\right|_{V_{0}}=\left.u_{2}\right|_{V_{0}}=0, & \end{cases}
$$

where $V$ stands for the Sierpiński gasket, $V_{0}$ is its intrinsic boundary, $\Delta$ denotes the weak Laplacian on $V$ and $\lambda$ is a positive real parameter.

We assume that $F: \mathbb{R}^{2} \rightarrow \mathbb{R}$ is a $C^{1}$-function such that $F(0,0)=0$, and $F_{u_{i}}$ denotes the partial derivative of $F$ with respect to $u_{i}$. Finally, the variable potentials $a_{1}, a_{2}, g: V \rightarrow \mathbb{R}$ satisfy the following conditions:

$\left(\mathrm{h}_{1}\right) a_{i} \in L^{1}(V, \mu)$ and $a_{i} \leq 0(i=1,2)$ almost everywhere in $V$;

$\left(\mathrm{h}_{2}\right) g \in C(V)$ with $g \leq 0$ such that the restriction of $g$ to every open subset of $V$ is not identically zero.

Here $\mu$ denotes the restriction to $V$ of the normalized $\frac{\log N}{\log 2}$-dimensional Hausdorff measure on $V$, so that $\mu(V)=1$; see, for more details, the recent work [18].

The nonlinear problem $\left(S_{\lambda}\right)$ is closely related to physical phenomena such as reactiondiffusion problems and elastic properties of fractal media and flow through fractal regions. There is an extensive theory for the study of nonlinear elliptic equations $\left(S_{\lambda}\right)$ on classical domains, that is, on open sets of $\mathbb{R}^{N}$, using Sobolev spaces and Sobolev embedding theorems etc. (see $[3-5,20,23,29]$ ). Many solvability conditions are given, such as the conditions in the fibering method introduced by Pohozaev and the study of the Nehari manifold for some classes of quasilinear elliptic systems involving a pair of Laplacian operators (see [16, 47]).

A natural question arises of how to establish an appropriate framework to cope with $\left(S_{\lambda}\right)$ on fractal domains. Here we work on a specific fractal, the Sierpiński gasket $V$ in $\mathbb{R}^{N-1}(N \geq 2)$, which is typical one of the more general class of post-critically finite fractals.

Over the years, the Sierpiński gasket showed to be extraordinarily useful in representing roughness in nature and man's works. We refer to [44] for an elementary introduction to this subject and to [46] for important applications to differential equations on fractals. We also refer to the excellent monograph [19] for a thorough introduction to relevant applications of the nonlinear analysis.

Moreover, this geometrical object represents one of the most familiar examples of fractal domains and it gives insight into the turbulence of fluids. According to [33], this notion was introduced by Mandelbrot [37] in 1977 to design a class of mathematical objects which are not collections of smooth components.

The importance of fractals is given by their utility in physics, chemistry or biology. Moreover, the study of the Laplacian on fractals originated in physics literature, where the so-called spectral decimation method was developed in [1, 40-41]. For completeness we recall that the Laplacian on the Sierpiński gasket was first constructed as the generator of a diffusion process (see $[28,35])$.

Here we are interested in the existence of infinitely many solutions for a system of gradienttype $\left(S_{\lambda}\right)$ by using variational methods. In our main results, just requiring an oscillating behavior of the term $F$ either at zero or at infinity, we prove, without symmetry assumptions, that the problem $\left(S_{\lambda}\right)$ admits a sequence of pairwise distinct weak solutions, see Theorems 3.1-3.2 below. Our method strongly relies on the following critical points theorem, which is a more precise version of [42, Theorem 2.5].

Theorem 1.1 (see [9, Theorem 2.1]) Let $E$ be a reflexive real Banach space, and let $\Phi, \Psi: E \rightarrow \mathbb{R}$ be two Gâteaux differentiable functionals such that $\Phi$ is strongly continuous, sequentially weakly lower semi-continuous and coercive, and $\Psi$ is sequentially weakly upper 
semi-continuous. For every $r>\inf _{E} \Phi$, put

$$
\varphi(r):=\inf _{u \in \Phi^{-1}(]-\infty, r[)} \frac{\sup _{v \in \Phi^{-1}(]-\infty, r[)} \Psi(v)-\Psi(u)}{r-\Phi(u)}
$$

and

$$
\gamma:=\liminf _{r \rightarrow+\infty} \varphi(r), \quad \delta:=\liminf _{r \rightarrow\left(\inf _{E} \Phi\right)^{+}} \varphi(r)
$$

Then, one has

(a) If $\gamma<+\infty$ then, for each $\lambda \in] 0, \frac{1}{\gamma}[$, the following alternative holds: Either

$\left(\mathrm{a}_{1}\right) \quad J_{\lambda}:=\Phi-\lambda \Psi$ possesses a global minimum,

or

$\left(\mathrm{a}_{2}\right)$ there exists a sequence $\left\{w_{n}\right\}$ of critical points (local minima) of $J_{\lambda}$ such that $\lim _{n \rightarrow \infty} \Phi\left(w_{n}\right)=+\infty$.

(b) If $\delta<+\infty$ then, for each $\lambda \in] 0, \frac{1}{\delta}[$, the following alternative holds:

Either

$\left(\mathrm{b}_{1}\right)$ there exists a global minimum of $\Phi$ which is a local minimum of $J_{\lambda}$,

or

$\left(\mathrm{b}_{2}\right)$ there exists a sequence $\left\{w_{n}\right\}$ of pairwise distinct critical points (local minima) of $J_{\lambda}$ which weakly converges to a global minimum of $\Phi$, with $\lim _{n \rightarrow \infty} \Phi\left(w_{n}\right)=\inf _{w \in E} \Phi(w)$.

The above theorem assures the existence of a sequence of pairwise distinct critical points for Gâteaux differentiable functionals under the assumptions that, when we consider the energy functional associated to $\left(S_{\lambda}\right)$, are satisfied just assuming an appropriate oscillating behavior on the potential of the nonlinearity either at infinity or at zero (see Theorems 3.1-3.2).

This method has been used successfully to prove, in the context of certain Sobolev spaces, the existence of infinitely many solutions for Dirichlet and Neumann equations (see [7-8, 10, 12-13, 21-22]). In the present paper, we are able to show that the methods used in [11] can be successfully adapted to prove the existence of infinitely many (weak) solutions also for nonlinear elliptic systems of the gradient-type on fractal domains.

Many technical difficulties are overcome in our approach by using suitable analytic properties arising from the geometry of the Sierpiński gasket (see Remark 3.1). For instance, a careful analysis of the normalized measure $\mu$, proved by Breckner, Rădulescu and Varga in [17], will be essential to proving that, under certain hypotheses, the energy functional associated to $\left(S_{\lambda}\right)$ is unbounded from below (see the proof of Theorem 3.1).

In Corollaries 3.1-3.2, we discuss the case of a two-parametric Dirichlet system $\left(S_{\lambda, \mu}\right)$, showing the existence of well-determined open interval of parameters $\lambda$ and $\mu$ for which problem $\left(S_{\lambda, \mu}\right)$ admits infinitely many solutions (see [22] for related topics).

Further, existence results, analogous of Theorems 3.1-3.2, for sign-changing potential, can be obtained requiring an additional hypothesis on the nonlinearity either at infinity or at zero (see Remarks 3.2-3.3).

Finally, we observe that if the data of our problems are sufficiently regular, every weak solution is a strong solution (see Remark 2.2 and Lemma 2.16 of Falconer and Hu [26]).

The plan of the paper is as follows. In Section 2 we recall some basic facts on suitable Sobolev spaces associated to the Sierpiński gasket. Successively, Section 3 is devoted to the main theorems. A direct application is presented in the final section of this paper. 
We cite the very recent monograph [34] as a general reference for the basic notions used in the paper. Finally, we denote by $\mathbb{N}$ the set of natural numbers $\{0,1,2, \cdots\}$ and by $|\cdot|$ the Euclidian norm on the space $\mathbb{R}^{N}$.

\section{Preliminaries}

The Sierpiński gasket has the origin in a paper by Sierpiński [43] and in a very simple manner can be described as a subset of the plane obtained from an equilateral triangle by removing the open middle inscribed equilateral triangle of $\frac{1}{4}$ of the area, removing the corresponding open triangle from each of the three constituent triangles and continuing in this way. This fractal can also be obtained as the closure of the set of vertices arising in this construction.

Let $V$ be the Sierpiński gasket in $\mathbb{R}^{N-1}(N \geq 2)$ of an intrinsic boundary $V_{0}$. For a direct construction and more details on this topics, see the paper [18].

Denote by $C(V)$ the space of real-valued continuous functions on $V$ and

$$
C_{0}(V):=\left\{u \in C(V) ;\left.u\right|_{V_{0}}=0\right\} .
$$

The spaces $C(V)$ and $C_{0}(V)$ are endowed with the usual supremum norm $\|\cdot\|_{\infty}$. For a function $u: V \rightarrow \mathbb{R}$ and for $m \in \mathbb{N}$, let

$$
W_{m}(u)=\left(\frac{N+2}{N}\right)^{m} \sum_{\substack{x, y \in V_{m} \\|x-y|=2^{-m}}}(u(x)-u(y))^{2} .
$$

We have $W_{m}(u) \leq W_{m+1}(u)$ for very natural $m$. So we can put

$$
W(u)=\lim _{m \rightarrow \infty} W_{m}(u) .
$$

Define

$$
H_{0}^{1}(V):=\left\{u \in C_{0}(V) ; W(u)<\infty\right\} .
$$

It turns out that $H_{0}^{1}(V)$ is a dense linear subset of $L^{2}(V, \mu)$ equipped with the $\|\cdot\|_{2}$ norm. We now endow $H_{0}^{1}(V)$ with the norm

$$
\|u\|=\sqrt{W(u)} .
$$

In fact, there is an inner product defining this norm: for $u, v \in H_{0}^{1}(V)$ and $m \in \mathbb{N}$, let

$$
\mathcal{W}_{m}(u, v)=\left(\frac{N+2}{N}\right)^{m} \sum_{\substack{x, y \in V_{m} \\|x-y|=2^{-m}}}(u(x)-u(y))(v(x)-v(y)) .
$$

Kigami's idea (see [32-33]) is to approximate the fractal from within by a sequence of finite graphs. The Laplace operator on the fractal is then the renormalized limit of graph Laplacians. This construction is described in what follows. We first define

$$
\mathcal{W}(u, v)=\lim _{m \rightarrow \infty} \mathcal{W}_{m}(u, v)
$$

Then $\mathcal{W}(u, v) \in \mathbb{R}$ and the space $H_{0}^{1}(V)$, equipped with the inner product $\mathcal{W}$, which induces the norm $\|\cdot\|$, becomes a real Hilbert space.

Moreover,

$$
\|u\|_{\infty} \leq(2 N+3)\|u\| \quad \text { for every } u \in H_{0}^{1}(V)
$$


and the embedding

$$
\left(H_{0}^{1}(V),\|\cdot\|\right) \hookrightarrow\left(C_{0}(V),\|\cdot\|_{\infty}\right)
$$

is compact. We refer to [27] for further details.

Remark 2.1 As pointed out by Falconer and Hu [26], we just observe that if $a \in L^{1}(V)$ and $a \leq 0$ in $V$, then from (2.3), the norm

$$
\|u\|_{*}:=\left(\mathcal{W}(u, u)-\int_{V} a(x) u^{2} \mathrm{~d} \mu\right)^{\frac{1}{2}}
$$

is equivalent to $\sqrt{W(u)}$ in $H_{0}^{1}(V)$.

We now state a useful property of the space $H_{0}^{1}(V)$ which shows, together with the facts that $\left(H_{0}^{1}(V),\|\cdot\|\right)$ is a Hilbert space and $H_{0}^{1}(V)$ is dense in $L^{2}(V, \mu)$, that $\mathcal{W}$ is a Dirichlet form on $L^{2}(V, \mu)$.

Lemma 2.1 Let $h: \mathbb{R} \rightarrow \mathbb{R}$ be a Lipschitz mapping with the Lipschitz constant $L \geq 0$ such that $h(0)=0$. Then, for every $u \in H_{0}^{1}(V)$, we have $h \circ u \in H_{0}^{1}(V)$ and $\|h \circ u\| \leq L\|u\|$.

Proof It is clear that $h \circ u \in C_{0}(V)$. For every $m \in \mathbb{N}$, by (2.1) and the Lipschitz property of $h$, we have that

$$
W_{m}(h \circ u) \leq L^{2} W_{m}(u) .
$$

Hence $W(h \circ u) \leq L^{2} W(u)$, according to (2.2). Thus $h \circ u \in H_{0}^{1}(V)$ and $\|h \circ u\| \leq L\|u\|$.

Following Falconer and $\mathrm{Hu}[26]$, a standard way we can define in a linear self-adjoint operator $\Delta: Z \rightarrow L^{2}(V, \mu)$, where $Z$ is a linear subset of $H_{0}^{1}(V)$, which is dense in $L^{2}(V, \mu)$ (and dense also in $\left.\left(H_{0}^{1}(V),\|\cdot\|\right)\right)$, such that

$$
-\mathcal{W}(u, v)=\int_{V} \Delta u \cdot v \mathrm{~d} \mu \quad \text { for every }(u, v) \in Z \times H_{0}^{1}(V) .
$$

The operator $\Delta$ is called a weak Laplacian on $V$.

Precisely, let $H^{-1}(V)$ be the closure of $L^{2}(V, \mu)$ with respect to the pre-norm

$$
\|u\|_{-1}=\sup _{\substack{h \in H_{0}^{1}(V) \\\|h\|=1}}|\langle u, h\rangle|
$$

where

$$
\langle v, h\rangle=\int_{V} v h \mathrm{~d} \mu,
$$

$v \in L^{2}(V, \mu)$ and $h \in H_{0}^{1}(V)$. Then $H^{-1}(V)$ is a Hilbert space, and the relation

$$
-\mathcal{W}(u, v)=\langle\Delta u, v\rangle, \quad \forall v \in H_{0}^{1}(V)
$$

uniquely defines a function $\Delta u \in H^{-1}(V)$ for every $u \in H_{0}^{1}(V)$.

Fix $\lambda>0$. We say that a function $\left(u_{1}, u_{2}\right) \in H_{0}^{1}(V) \times H_{0}^{1}(V)$ is called a weak solution of $\left(S_{\lambda}\right)$ if

$$
\sum_{i=1}^{2}\left[\left(\mathcal{W}\left(u_{i}, v_{i}\right)-\int_{V} a_{i}(x) u_{i}(x) v_{i}(x) \mathrm{d} \mu\right)+\lambda \int_{V} g(x) F_{u_{i}}\left(u_{1}(x), u_{2}(x)\right) v_{i}(x) \mathrm{d} \mu\right]=0
$$

for every $\left(v_{1}, v_{2}\right) \in H_{0}^{1}(V) \times H_{0}^{1}(V)$. 
While we mainly work with the weak Laplacian, there is also a directly defined version. We say that $\Delta_{s}$ is the standard Laplacian of $u$ if $\Delta_{s} u: V \rightarrow \mathbb{R}$ is continuous and

$$
\lim _{m \rightarrow \infty} \sup _{x \in V \backslash V_{0}}\left|(N+2)^{m}\left(H_{m} u\right)(x)-\Delta_{s} u(x)\right|=0,
$$

where

$$
\left(H_{m} u\right)(x):=\sum_{\substack{y \in V_{m} \\|x-y|=2^{-m}}}(u(y)-u(x))
$$

for $x \in V_{m}$.

We say that $\left(u_{1}, u_{2}\right) \in C_{0}(V) \times C_{0}(V)$ is a strong solution of $\left(S_{\lambda}\right)$ if $\Delta_{s} u_{1}$ and $\Delta_{s} u_{2}$ exist and are continuous for all $x \in V \backslash V_{0}$, in addition to

$$
\begin{cases}\Delta_{s} u_{1}(x)+a_{1}(x) u_{1}(x)=\lambda g(x) F_{u_{1}}\left(u_{1}(x), u_{2}(x)\right), & x \in V \backslash V_{0}, \\ \Delta_{s} u_{2}(x)+a_{2}(x) u_{2}(x)=\lambda g(x) F_{u_{2}}\left(u_{1}(x), u_{2}(x)\right), & x \in V \backslash V_{0}, \\ \left.u_{1}\right|_{V_{0}}=\left.u_{2}\right|_{V_{0}}=0 . & \end{cases}
$$

The existence of the standard Laplacian of a function $u \in H_{0}^{1}(V)$ implies the existence of the weak Laplacian $\Delta$, see, for completeness, Falconer and $\mathrm{Hu}[26]$.

Remark 2.2 If $a_{1}, a_{2} \in C(V)$, arguing as in Lemma 2.16 of [26], it follows that every weak solution of the problem $\left(S_{\lambda}\right)$ is also a strong solution.

\section{Main Results}

In this section, we assume that $F: \mathbb{R}^{2} \rightarrow \mathbb{R}$ is a $C^{1}$-function such that $F(0,0)=0$ and $F_{u_{i}}$ denotes the partial derivative of $F$ with respect to $u_{i}$. Moreover, the variable potentials $a_{1}, a_{2}, g: V \rightarrow \mathbb{R}$ satisfy the following conditions:

$\left(\mathrm{h}_{1}\right) a_{i} \in L^{1}(V, \mu)$ and $a_{i} \leq 0(i=1,2)$ almost everywhere in $V$;

$\left(\mathrm{h}_{2}\right) g \in C(V)$ with $g \leq 0$ such that the restriction of $g$ to every open subset of $V$ is not identically zero.

For every $\xi>0$, set

$$
Q(\xi):=\left\{\left(t_{1}, t_{2}\right) \in \mathbb{R}^{2}: \sum_{i=1}^{2}\left|t_{i}\right| \leq \xi\right\}
$$

and

$$
\mathbb{R}_{+}^{2}=\left\{\left(t_{1}, t_{2}\right) \in \mathbb{R}^{2}: t_{i} \geq 0, \forall i=1,2\right\} .
$$

With the above notations, we have the following existence result in the case of nonlinearities oscillating at infinity.

Theorem 3.1 Let $F$ be nonnegative on $\mathbb{R}_{+}^{2}$. Further, assume that

$$
A_{\infty}:=\liminf _{\xi \rightarrow+\infty} \frac{\max _{\left(t_{1}, t_{2}\right) \in Q(\xi)} F\left(t_{1}, t_{2}\right)}{\xi^{2}}<+\infty \quad\left(F_{\infty}^{1}\right)
$$

and

$$
\begin{aligned}
& \limsup _{\substack{\left(t_{1}, t_{2}\right) \rightarrow \infty \\
\left(t_{1}, t_{2}\right) \in \mathbb{R}_{+}^{2}}} \\
& \sum_{i=1}^{2} t_{i}^{2}
\end{aligned}
$$


Then, for every

$$
\lambda \in] 0,-\frac{1}{8(2 N+3)^{2}\left(\int_{V} g(x) \mathrm{d} \mu\right) A_{\infty}}[,
$$

there exists an unbounded sequence of weak solutions of the problem $\left(S_{\lambda}\right)$.

Proof Let us define the functionals $\Phi, \Psi: E \rightarrow \mathbb{R}$ by

$$
\Phi\left(u_{1}, u_{2}\right)=\frac{1}{2} \sum_{i=1}^{2}\left(\left\|u_{i}\right\|^{2}-\int_{V} a_{i}(x) u_{i}(x)^{2} \mathrm{~d} \mu\right)
$$

and

$$
\Psi\left(u_{1}, u_{2}\right)=-\int_{V} g(x) F\left(u_{1}(x), u_{2}(x)\right) \mathrm{d} \mu,
$$

respectively, where the product space $E:=H_{0}^{1}(V) \times H_{0}^{1}(V)$ is endowed by the norm

$$
\left\|\left(u_{1}, u_{2}\right)\right\|:=\sum_{i=1}^{2}\left(W\left(u_{i}\right)-\int_{V} a_{i}(x) u_{i}(x)^{2} \mathrm{~d} \mu\right)^{\frac{1}{2}}
$$

for every $\left(u_{1}, u_{2}\right) \in E$. Now, in order to achieved our goal, fix $\lambda$ as in the conclusion. Clearly, with the above notations, set $J_{\lambda}:=\Phi-\lambda \Psi$. First of all, we observe that the functional $J_{\lambda} \in C^{1}(E, \mathbb{R})$.

Further, fixing $\left(u_{1}, u_{2}\right) \in E$, one has

$$
\begin{aligned}
J_{\lambda}^{\prime}\left(u_{1}, u_{2}\right)\left(v_{1}, v_{2}\right)= & \sum_{i=1}^{2}\left(\mathcal{W}\left(u_{i}, v_{i}\right)-\int_{V} a_{i}(x) u_{i}(x) v_{i}(x) \mathrm{d} \mu\right) \\
& +\lambda \sum_{i=1}^{2} \int_{V} g(x) F_{u_{i}}\left(u_{1}(x), u_{2}(x)\right) v_{i}(x) \mathrm{d} \mu
\end{aligned}
$$

for each $\left(v_{1}, v_{2}\right) \in E$. In particular, $\left(u_{1}, u_{2}\right) \in E$ is a weak solution of the problem $\left(S_{\lambda}\right)$ if and only if $\left(u_{1}, u_{2}\right)$ is a critical point of $J_{\lambda}$. Clearly, $\Phi$ is obviously coercive. Moreover, standard computations ensure that the functionals $\Phi$ and $\Psi$ are sequentially weakly lower semi-continuous on $E$. Hence, we seek for weak solutions of problem $\left(S_{\lambda}\right)$ by applying part (a) of Theorem 1.1.

Now let us verify that $\gamma<+\infty$. For our purpose, let $\left\{c_{n}\right\}$ be a sequence (of positive numbers), such that $\lim _{n \rightarrow \infty} c_{n}=+\infty$ and

$$
\lim _{n \rightarrow \infty} \frac{\max _{\left(t_{1}, t_{2}\right) \in Q\left(c_{n}\right)} F\left(t_{1}, t_{2}\right)}{c_{n}^{2}}=A_{\infty}
$$

Put $r_{n}:=\frac{c_{n}^{2}}{8(2 N+3)^{2}}$ for every $n \in \mathbb{N}$. Due to the compact embedding of $H_{0}^{1}(V)$ into $C_{0}(V)$, we have

$$
\left\{\left(v_{1}, v_{2}\right) \in E: \Phi\left(v_{1}, v_{2}\right)<r_{n}\right\} \subseteq\left\{\left(v_{1}, v_{2}\right) \in E: \sum_{i=1}^{2}\left|v_{i}(x)\right| \leq c_{n}, \forall x \in V\right\} .
$$

Indeed, taking into account (2.3), for every $v_{i} \in H_{0}^{1}(V)(i=1,2)$, one has

$$
\max _{x \in V}\left|v_{i}(x)\right|^{2} \leq(2 N+3)^{2}\left\|v_{i}\right\|^{2} .
$$


Consequently,

$$
\max _{x \in V} \sum_{i=1}^{2} \frac{\left|v_{i}(x)\right|^{2}}{2} \leq(2 N+3)^{2}\left(\sum_{i=1}^{2} \frac{\left\|v_{i}\right\|^{2}}{2}\right)=(2 N+3)^{2} \Phi\left(v_{1}, v_{2}\right) .
$$

At this point, from the above inequality, if $\Phi\left(v_{1}, v_{2}\right)<r_{n}$, a direct computation ensures that $\sum_{i=1}^{m}\left|v_{i}(x)\right| \leq c_{n}$, for every $x \in V$.

Therefore, taking into account that $0_{E} \in \Phi^{-1}(]-\infty, r_{n}[)$ for every $n \in \mathbb{N}$, since $F(0,0)=0$, one has

$$
\begin{array}{r}
\varphi\left(r_{n}\right)=\inf _{\sum_{i=1}^{2} \frac{\left\|u_{i}\right\|^{2}}{2}<r_{n}} \frac{\sum_{i=1}^{2} \frac{\left\|v_{i}\right\|^{2}}{2}<r_{n}}{r_{n}-\left(\sum_{i=1}^{2} \frac{\left\|u_{i}\right\|^{2}}{2}\right)} \\
\left.\leq \frac{\sum_{i=1}^{2} \frac{\left\|v_{i}\right\|^{2}}{p_{i}}<r_{n}}{\leq} \int_{V}-g(x) F\left(v_{1}(x), v_{2}\right)-\Psi\left(u_{1}, u_{2}\right)\right) \mathrm{d} x \\
\leq-8(2 N+3)^{2}\left(\int_{V} g(x) \mathrm{d} \mu\right) \frac{\left(t_{1}, t_{2}\right) \in Q\left(c_{n}\right)}{c_{n}^{2}} F\left(t_{1}, t_{2}\right)
\end{array} .
$$

Thus, bearing in mind that

$$
\liminf _{\xi \rightarrow+\infty} \frac{\max _{\left(t_{1}, t_{2}\right) \in Q(\xi)} F\left(t_{1}, t_{2}\right)}{\xi^{2}}<+\infty
$$

we deduce

$$
\gamma \leq \liminf _{n \rightarrow \infty} \varphi\left(r_{n}\right) \leq-8(2 N+3)^{2}\left(\int_{V} g(x) \mathrm{d} \mu\right) \liminf _{\xi \rightarrow+\infty} \frac{\max _{\left(t_{1}, t_{2}\right) \in Q(\xi)} F\left(t_{1}, t_{2}\right)}{\xi^{2}}<+\infty .
$$

Let us verify that the functional $J_{\lambda}$ is unbounded from below. For our goal, fix a function $u \in H_{0}^{1}(V)$ such that there is an element $x_{0} \in V$ with $u\left(x_{0}\right)>1$. It follows that

$$
D:=\{x \in V ; u(x)>1\}
$$

is a non-empty open (from the continuity of $u$ ) subset of $V$. Moreover, from relation (2.1) in [18], one has $\mu(D)>0$. Define $h: \mathbb{R} \rightarrow \mathbb{R}$ as follows

$$
h(t)=|\min \{t, 1\}|
$$

for every $t \in \mathbb{R}$.

Then $h(0)=0$, and $h$ is a Lipschitz function whose Lipschitz constant $L$ is equal to 1 . Hence, by using Lemma 2.1, it follows that $v:=h \circ u \in H_{0}^{1}(V)$. Moreover, $v(x)=1$ for every $x \in D$, and $0 \leq v(x) \leq 1$ for every $x \in V$. As the condition $\left(F_{\infty}^{1}\right)$ holds, there exist two positive real sequences $\left\{\xi_{1, n}\right\}$ and $\left\{\xi_{2, n}\right\}$ such that

$$
\lim _{n \rightarrow \infty} \sqrt{\sum_{i=1}^{2} \xi_{i, n}^{2}}=+\infty
$$


and

$$
\lim _{n \rightarrow \infty} \frac{F\left(\xi_{1, n}, \xi_{2, n}\right)}{\sum_{i=1}^{2} \xi_{i, n}^{2}}=+\infty .
$$

Consider the sequence of functions $\left\{\left(\xi_{1, n} v, \xi_{2, n} v\right)\right\} \subset E$. Thus

$$
\begin{aligned}
J_{\lambda}\left(\xi_{1, n} v, \xi_{2, n} v\right)= & \frac{\xi_{1, n}^{2}}{2}\|v\|^{2}-\frac{\xi_{1, n}^{2}}{2} \int_{V} a_{1}(x) v(x)^{2} \mathrm{~d} \mu \\
& +\frac{\xi_{2, n}^{2}}{2}\|v\|^{2}-\frac{\xi_{2, n}^{2}}{2} \int_{V} a_{2}(x) v(x)^{2} \mathrm{~d} \mu \\
& +\lambda \int_{V} g(x) F\left(\xi_{1, n} v(x), \xi_{2, n} v(x)\right) \mathrm{d} \mu .
\end{aligned}
$$

Now, since $F$ and $g$ are respectively nonnegative (on $\mathbb{R}_{+}^{2}$ ) and nonpositive (on $V$ ), it follows that

$$
\begin{aligned}
& \int_{V} g(x) F\left(\xi_{1, n} v(x), \xi_{2, n} v(x)\right) \mathrm{d} \mu \\
= & F\left(\xi_{1, n}, \xi_{2, n}\right) \int_{D} g(x) \mathrm{d} \mu+\int_{V \backslash D} g(x) F\left(\xi_{1, n} v(x), \xi_{2, n} v(x)\right) \mathrm{d} \mu \\
\leq & F\left(\xi_{1, n}, \xi_{2, n}\right) \int_{D} g(x) \mathrm{d} \mu .
\end{aligned}
$$

Further, putting

$$
\vartheta(v):=\max \left\{\|v\|^{2}-\int_{V} a_{1}(x) v(x)^{2} \mathrm{~d} \mu,\|v\|^{2}-\int_{V} a_{2}(x) v(x)^{2} \mathrm{~d} \mu\right\},
$$

from (3.2)-(3.3) one has

$$
J_{\lambda}\left(\xi_{1, n} v, \xi_{2, n} v\right) \leq\left(\frac{1}{2} \sum_{i=1}^{2} \xi_{i, n}^{2}\right) \vartheta(v)+\lambda F\left(\xi_{1, n}, \xi_{2, n}\right) \int_{D} g(x) \mathrm{d} \mu,
$$

which means

$$
\frac{J_{\lambda}\left(\xi_{1, n} v, \xi_{2, n} v\right)}{\sum_{i=1}^{2} \xi_{i, n}^{2}} \leq \frac{\vartheta(v)}{2}+\lambda \frac{F\left(\xi_{1, n}, \xi_{2, n}\right)}{\sum_{i=1}^{2} \xi_{i, n}^{2}} \int_{D} g(x) \mathrm{d} \mu .
$$

At this point, since from (3.1) one has

$$
\lim _{n \rightarrow \infty} \frac{F\left(\xi_{1, n}, \xi_{2, n}\right)}{\sum_{i=1}^{2} \xi_{i, n}^{2}} \int_{D} g(x) \mathrm{d} \mu=-\infty
$$

(note that $\int_{D} g(x) \mathrm{d} \mu<0$ ) inequality (3.4) implies that

$$
\lim _{n \rightarrow \infty} \frac{J_{\lambda}\left(\xi_{1, n} v, \xi_{2, n} v\right)}{\sum_{i=1}^{2} \xi_{i, n}^{2}}=-\infty
$$


In conclusion

$$
\lim _{n \rightarrow \infty} J_{\lambda}\left(\xi_{1, n} v, \xi_{2, n} v\right)=-\infty
$$

and the functional $J_{\lambda}$ is unbounded from below. Applying Theorem 1.1 we deduce that the functional $J_{\lambda}$ admits a sequence of critical points which is unbounded in $E$. Hence, our claim is proved, and the conclusion is achieved.

Remark 3.1 It is worth noting that the above statements and the proof of our method are related to the corresponding ones in [11]. Clearly, the abstract framework introduced in the above mentioned paper is adaptable to our context by using the geometric and analytic properties of the Sierpiński fractal as the Sobolev-type inequality

$$
\sup _{x, y \in V_{*}} \frac{|u(x)-u(y)|}{|x-y|^{\sigma}} \leq(2 N+3) \sqrt{W(u)},
$$

where

$$
\sigma:=\frac{\log \left(\frac{N+2}{N}\right)}{2 \log 2},
$$

(see, for more details in [26, Lemma 2.4]).

We note that the estimate (3.5) allows all $u: V_{*} \rightarrow \mathbb{R}$ of finite energy to have a continuous extension to $V$. Moreover, through (3.5) and by using the Ascoli-Arzéla theorem, the compact embedding (2.4) is achieved.

Remark 3.2 We explicitly observe that, exploiting the proof of Theorem 3.1, one can see that the statements of our result are still true also for sign-changing functions $F: \mathbb{R}^{2} \rightarrow \mathbb{R}$ that satisfy assumptions $\left(F_{\infty}^{1}\right)$ and $\left(F_{\infty}^{2}\right)$ in addition to

$$
\liminf _{\left(t_{1}, t_{2}\right) \rightarrow \infty} \frac{F\left(t_{1}, t_{2}\right)}{\sum_{i=1}^{2} t_{i}^{2}}>-\infty .
$$

Indeed, if

$$
\liminf _{\xi \rightarrow+\infty} \frac{\max _{\left(t_{1}, t_{2}\right) \in Q(\xi)} F\left(t_{1}, t_{2}\right)}{\xi^{2}}<+\infty,
$$

one has that $\gamma<+\infty$. On the other hand, consider the sequence of functions $\left\{\left(\xi_{1, n} v, \xi_{2, n} v\right)\right\} \subset$ $E$ as in the proof of Theorem 3.1. From

$$
\liminf _{\left(t_{1}, t_{2}\right) \rightarrow \infty} \frac{F\left(t_{1}, t_{2}\right)}{\sum_{i=1}^{2} t_{i}^{2}}>-\infty,
$$

there exist $\varrho, k>0$ such that

$$
\frac{F\left(t_{1}, t_{2}\right)}{\sum_{i=1}^{2} t_{i}^{2}} \geq-k, \quad \text { if } \sum_{i=1}^{2} t_{i}^{2}>\varrho .
$$

Moreover, one has

$$
\begin{aligned}
J_{\lambda}\left(\xi_{1, n} v, \xi_{2, n} v\right)= & \frac{\xi_{1, n}^{2}}{2}\|v\|^{2}-\frac{\xi_{1, n}^{2}}{2} \int_{V} a_{1}(x) v(x)^{2} \mathrm{~d} \mu+\frac{\xi_{2, n}^{2}}{2}\|v\|^{2}-\frac{\xi_{2, n}^{2}}{2} \int_{V} a_{2}(x) v(x)^{2} \mathrm{~d} \mu \\
& +\lambda F\left(\xi_{1, n}, \xi_{2, n}\right) \int_{D} g(x) \mathrm{d} \mu+\lambda \int_{V \backslash D} g(x) F\left(\xi_{1, n} v(x), \xi_{2, n} v(x)\right) \mathrm{d} \mu
\end{aligned}
$$


for every $n \in \mathbb{N}$.

Moreover

$$
\begin{aligned}
\int_{V \backslash D} g(x) F\left(\xi_{1, n} v(x), \xi_{2, n} v(x)\right) \mathrm{d} \mu= & \int_{G_{\varrho} \cap(V \backslash D)} g(x) F\left(\xi_{1, n} v(x), \xi_{2, n} v(x)\right) \mathrm{d} \mu \\
& +\int_{G^{e} \cap(V \backslash D)} g(x) F\left(\xi_{1, n} v(x), \xi_{2, n} v(x)\right) \mathrm{d} \mu,
\end{aligned}
$$

where

$$
G_{\varrho}:=\left\{x \in V: 0 \leq\left(\sum_{i=1}^{2} \xi_{i, n}^{2}\right) v(x)^{2} \leq \varrho\right\}
$$

and

$$
G^{\varrho}:=\left\{x \in V:\left(\sum_{i=1}^{2} \xi_{i, n}^{2}\right) v(x)^{2}>\varrho\right\} .
$$

Now, by using the mean value theorem, it follows that

$$
\int_{G_{\varrho} \cap(V \backslash D)} g(x) F\left(\xi_{1, n} v(x), \xi_{2, n} v(x)\right) \mathrm{d} \mu \leq C,
$$

where

$$
C:=2\|g\|_{\infty} \max _{\left(t_{1}, t_{2}\right) \in[0, \sqrt{\varrho}]^{2}}\left|\nabla F\left(t_{1}, t_{2}\right)\right| \varrho .
$$

Then, relations (3.6) and (3.7) yield

$$
\begin{aligned}
J_{\lambda}\left(\xi_{1, n} v, \xi_{2, n} v\right) \leq & \left(\frac{1}{2} \sum_{i=1}^{2} \xi_{i, n}^{2}\right) \vartheta(v)+\lambda F\left(\xi_{1, n}, \xi_{2, n}\right) \int_{D} g(x) \mathrm{d} \mu \\
& +2 \lambda\|g\|_{\infty} \max _{\left(t_{1}, t_{2}\right) \in[0, \sqrt{\varrho}]^{2}}\left|\nabla F\left(t_{1}, t_{2}\right)\right| \varrho \\
& -k \lambda\left(\sum_{i=1}^{2} \xi_{i, n}^{2}\right) \int_{V \backslash D} g(x) v^{2}(x) \mathrm{d} \mu
\end{aligned}
$$

for every $n \in \mathbb{N}$.

Thus, it is easy to see that the above inequality and our assumptions imply

$$
\lim _{n \rightarrow \infty} \frac{J_{\lambda}\left(\xi_{1, n} v, \xi_{2, n} v\right)}{\sum_{i=1}^{2} \xi_{i, n}^{2}}=-\infty .
$$

Then $J_{\lambda}$ is unbounded from below. The proof is attained from part (a) of Theorem 1.1.

Now, as a direct consequence of Theorem 3.1, we show that an appropriate oscillating behavior of the nonlinear term $F$, even under certain small perturbations, ensures again the existence of infinitely many solutions.

Denote by $\mathfrak{F}_{V}$ the set of functions $G: \mathbb{R}^{2} \rightarrow \mathbb{R}$ such that:

$\left(\mathrm{g}_{1}\right) \quad G$ is a $C^{1}$-function such that $G(0,0)=0$ and $G_{u_{i}}$ denotes the partial derivative of $G$ with respect to $u_{i}$;

$\left(\mathrm{g}_{2}\right) \quad G\left(t_{1}, t_{2}\right) \geq 0$ for every $\left(t_{1}, t_{2}\right) \in \mathbb{R}_{+}^{2}$.

We have the following existence result. 
Corollary 3.1 Under assumptions of Theorem 3.1, for each

$$
\lambda \in] 0,-\frac{1}{8(2 N+3)^{2}\left(\int_{V} g(x) \mathrm{d} \mu\right) A_{\infty}}[,
$$

for every $G \in \mathfrak{F}_{V}$ satisfying

$$
\left(\mathrm{g}_{\infty}\right) \quad G_{\infty}:=\lim _{\xi \rightarrow+\infty} \frac{\max _{\left(t_{1}, t_{2}\right) \in Q(\xi)} G\left(t_{1}, t_{2}\right)}{\xi^{2}}<+\infty
$$

and for every $\mu \in\left[0, \mu_{G, \lambda}[\right.$, where

$$
\mu_{G, \lambda}:=-\frac{1}{8(2 N+3)^{2}\left(\int_{V} g(x) \mathrm{d} \mu\right) G_{\infty}}\left(1+8 \lambda(2 N+3)^{2}\left(\int_{V} g(x) \mathrm{d} \mu\right) A_{\infty}\right),
$$

the problem (namely $\left.\left(S_{\lambda, \mu}\right)\right)$ given by

$$
\begin{cases}\Delta u_{1}(x)+a_{1}(x) u_{1}(x)=g(x)\left(\lambda F_{u_{1}}\left(u_{1}(x), u_{2}(x)\right)+\mu G_{u_{1}}\left(u_{1}(x), u_{2}(x)\right)\right), & x \in V \backslash V_{0}, \\ \Delta u_{2}(x)+a_{2}(x) u_{2}(x)=g(x)\left(\lambda F_{u_{2}}\left(u_{1}(x), u_{2}(x)\right)+\mu G_{u_{1}}\left(u_{1}(x), u_{2}(x)\right)\right), & x \in V \backslash V_{0}, \\ \left.u_{1}\right|_{V_{0}}=\left.u_{2}\right|_{V_{0}}=0, & \end{cases}
$$

admits a sequence of weak solutions which is unbounded in $H_{0}^{1}(V) \times H_{0}^{1}(V)$.

Proof Fix $\bar{\lambda} \in] 0, \lambda_{2}[$, where

$$
\lambda_{2}:=-\frac{1}{8(2 N+3)^{2}\left(\int_{V} g(x) \mathrm{d} \mu\right) A_{\infty}},
$$

and let $G \in \mathfrak{F}_{V}$ be a function satisfying hypothesis $\left(\mathrm{g}_{\infty}\right)$. In the non-perturbed case, that is $\mu=0$, the assertion is trivial. Otherwise, one has

$$
\mu_{G, \bar{\lambda}}:=-\frac{1}{8(2 N+3)^{2}\left(\int_{V} g(x) \mathrm{d} \mu\right) G_{\infty}}\left(1+8 \bar{\lambda}(2 N+3)^{2}\left(\int_{V} g(x) \mathrm{d} \mu\right) A_{\infty}\right)>0 .
$$

Take $0<\bar{\mu}<\mu_{G, \bar{\lambda}}$ and put

$$
\eta_{2}:=-\frac{1}{8(2 N+3)^{2}\left(\int_{V} g(x) \mathrm{d} \mu\right)\left(A_{\infty}+\frac{\bar{\mu}}{\bar{\lambda}} G_{\infty}\right)} .
$$

If $G_{\infty}=0$, clearly one has $\eta_{2}=\lambda_{2}$ and

$$
\left.\bar{\lambda} \in \Lambda^{\star}:=\right] 0, \eta_{2}[.
$$

Otherwise, if $G_{\infty} \neq 0$, from $\bar{\mu}<\mu_{g, \bar{\lambda}}$, it follows that

$$
-8(2 N+3)^{2}\left(\int_{V} g(x) \mathrm{d} \mu\right)\left(A_{\infty} \bar{\lambda}+G_{\infty} \bar{\mu}\right)<1,
$$

which means

$$
\bar{\lambda}<-\frac{1}{8(2 N+3)^{2}\left(\int_{V} g(x) \mathrm{d} \mu\right)\left(A_{\infty}+\frac{\bar{\mu}}{\bar{\lambda}} G_{\infty}\right)}=\eta_{2} .
$$


Hence, also in this case, one has

$$
\left.\bar{\lambda} \in \Lambda^{\star}:=\right] 0, \eta_{2}[.
$$

Now, put

$$
H\left(t_{1}, t_{2}\right):=F\left(t_{1}, t_{2}\right)+\frac{\bar{\mu}}{\bar{\lambda}} G\left(t_{1}, t_{2}\right)
$$

for every $t_{1}, t_{2} \in \mathbb{R}$.

Taking into account that the potential $G$ is a nonnegative function, we obtain

$$
\limsup _{\substack{\left(t_{1}, t_{2}\right) \rightarrow \infty \\
\left(t_{1}, t_{2}\right) \in \mathbb{R}_{+}^{2}}} \frac{H\left(t_{1}, t_{2}\right)}{\sum_{i=1}^{2} t_{i}^{2}} \geq \quad \begin{aligned}
& \limsup _{\left(t_{1}, t_{2}\right) \rightarrow \infty} \\
& \left(t_{1}, t_{2}\right) \in \mathbb{R}_{+}^{2} \\
& \sum_{i=1}^{2} t_{i}^{2}
\end{aligned} \frac{F\left(t_{1}, t_{2}\right)}{2}=+\infty
$$

and then

$$
\limsup _{\substack{\left(t_{1}, t_{2}\right) \rightarrow \infty \\\left(t_{1}, t_{2}\right) \in \mathbb{R}_{+}^{2}}} \frac{H\left(t_{1}, t_{2}\right)}{\sum_{i=1}^{2} t_{i}^{2}}=+\infty
$$

Moreover, since

$$
\frac{\max _{\left(t_{1}, t_{2}\right) \in Q(\xi)} H\left(t_{1}, t_{2}\right)}{\xi^{2}} \leq \frac{\max _{\left(t_{1}, t_{2}\right) \in Q(\xi)} F\left(t_{1}, t_{2}\right)}{\xi^{2}}+\frac{\bar{\mu}}{\bar{\lambda}} \frac{\max _{\left(t_{1}, t_{2}\right) \in Q(\xi)} G\left(t_{1}, t_{2}\right)}{\xi^{2}},
$$

taking into account hypothesis $\left(\mathrm{g}_{\infty}\right)$, it follows that

$$
\liminf _{\xi \rightarrow+\infty} \frac{\max _{\left(t_{1}, t_{2}\right) \in Q(\xi)} H\left(t_{1}, t_{2}\right)}{\xi^{2}} \leq \liminf _{\xi \rightarrow+\infty} \frac{\max _{\left(t_{1}, t_{2}\right) \in Q(\xi)} F\left(t_{1}, t_{2}\right)}{\xi^{2}}+\frac{\bar{\mu}}{\bar{\lambda}} G_{\infty}<+\infty .
$$

Hence, owing to

$$
\left.\bar{\lambda} \in \Lambda^{\star} \subseteq\right] 0,-\frac{1}{8(2 N+3)^{2}\left(\int_{V} g(x) \mathrm{d} \mu\right) H_{\infty}}[,
$$

where

$$
H_{\infty}:=\liminf _{\xi \rightarrow+\infty} \frac{\max _{\left(t_{1}, t_{2}\right) \in Q(\xi)} H\left(t_{1}, t_{2}\right)}{\xi^{2}},
$$

from Theorem 3.1, there exists an unbounded sequence of infinitely many solutions for problem $\left(S_{\bar{\lambda}, \bar{\mu}}\right)$. The proof is complete.

By the same method, applying part (b) instead of part (a) of Theorem 1.1, one can prove the analogue of Theorem 3.1 in presence of a smooth nonlinear term $F: \mathbb{R}^{2} \rightarrow \mathbb{R}$ with a suitable oscillating behavior at zero.

Theorem 3.2 Let $F$ be nonnegative on $\mathbb{R}_{+}^{2}$. Further, assume that

$$
A_{0}:=\liminf _{\xi \rightarrow 0^{+}} \frac{\max _{\left(t_{1}, t_{2}\right) \in Q(\xi)} F\left(t_{1}, t_{2}\right)}{\xi^{2}}<+\infty
$$

and

$$
\limsup _{\substack{\left(t_{1}, t_{2}\right) \rightarrow(0,0) \\\left(t_{1}, t_{2}\right) \in \mathbb{R}_{+}^{2}}} \frac{F\left(t_{1}, t_{2}\right)}{\sum_{i=1}^{2} t_{i}^{2}}=+\infty
$$


Then, for every

$$
\lambda \in] 0,-\frac{1}{8(2 N+3)^{2}\left(\int_{V} g(x) \mathrm{d} \mu\right) A_{\infty}}[,
$$

there exists a sequence $\left\{\left(u_{1, n}, u_{2, n}\right)\right\} \subset H_{0}^{1}(V) \times H_{0}^{1}(V)$ of pairwise distinct weak solutions of problem $\left(S_{\lambda}\right)$ such that $\lim _{n \rightarrow \infty}\left\|\left(u_{1, n}, u_{2, n}\right)\right\|=0$.

Proof The strategy of the proof is very similar to the previous one. Hence, in the sequel, we omit the details and we use the notations adopted in Theorem 3.1. Then, from hypothesis

$$
\liminf _{\xi \rightarrow 0^{+}} \frac{\max _{\left(t_{1}, t_{2}\right) \in Q(\xi)} F\left(t_{1}, t_{2}\right)}{\xi^{2}}<+\infty,
$$

by direct computations, it follows that $\delta:=\liminf _{r \rightarrow 0^{+}} \varphi(r)<+\infty$. On the other hand, by

$$
\limsup _{\substack{\left(t_{1}, t_{2}\right) \rightarrow(0,0) \\\left(t_{1}, t_{2}\right) \in \mathbb{R}_{+}^{2}}} \frac{F\left(t_{1}, t_{2}\right)}{\sum_{i=1}^{2} t_{i}^{2}}=+\infty
$$

there exist two positive real sequences, namely $\left\{\xi_{1, n}\right\}$ and $\left\{\xi_{2, n}\right\}$, such that

$$
\lim _{n \rightarrow \infty} \sqrt{\sum_{i=1}^{2} \xi_{i, n}^{2}}=0
$$

and

$$
\lim _{n \rightarrow \infty} \frac{F\left(\xi_{1, n}, \xi_{2, n}\right)}{\sum_{i=1}^{2} \xi_{i, n}^{2}}=+\infty .
$$

Now, consider the sequence of functions $\left\{\left(\xi_{1, n} v, \xi_{2, n} v\right)\right\} \subset E$. Arguing as in Theorem 3.1, we obtain

$$
\lim _{n \rightarrow \infty} \frac{J_{\lambda}\left(\xi_{1, n} v, \xi_{2, n} v\right)}{\sum_{i=1}^{2} \xi_{i, n}^{2}}=-\infty .
$$

Thus $J_{\lambda}\left(\xi_{1, n} v, \xi_{2, n} v\right)<0$ for every $n$ sufficiently large. Since $J_{\lambda}\left(0_{E}\right)=\Phi\left(0_{E}\right)-\lambda \Psi\left(0_{E}\right)=0$, the last inequality means that $0_{E}$ is not a local minimum of $J_{\lambda}$. Moreover, since $\Phi$ has $0_{E}$ as the unique global minimum, Theorem 1.1 ensures the existence of a sequence $\left\{\left(u_{1, n}, u_{2, n}\right)\right\} \subset E$ of pairwise distinct critical points of the functional $J_{\lambda}$, such that

$$
\lim _{n \rightarrow \infty} \sum_{i=1}^{2}\left(\left\|u_{i, n}\right\|^{2}-\int_{V} a_{i}(x) u_{i, n}(x)^{2} \mathrm{~d} \mu\right)=0 .
$$

Hence, one has that $\lim _{n \rightarrow \infty}\left\|\left(u_{1, n}, u_{2, n}\right)\right\|=0$. The proof is complete.

Remark 3.3 Theorem 3.2 also holds for sign-changing functions $F: \mathbb{R}^{2} \rightarrow \mathbb{R}$ that satisfy assumptions $\left(F_{0}^{1}\right)$ and $\left(F_{0}^{2}\right)$ in addition to

$$
\liminf _{\substack{\left(t_{1}, t_{2}\right) \rightarrow(0,0) \\\left(t_{1}, t_{2}\right) \in \mathbb{R}_{+}^{2}}} \frac{F\left(t_{1}, t_{2}\right)}{\sum_{i=1}^{2} t_{i}^{2}}>-\infty .
$$


The next result can be viewed as an analogue of Corollary 3.1.

Corollary 3.2 Under assumptions of Theorem 3.2, for each

$$
\lambda \in] 0,-\frac{1}{8(2 N+3)^{2}\left(\int_{V} g(x) \mathrm{d} \mu\right) A_{0}}[,
$$

for every $G \in \mathfrak{F}_{V}$ satisfying

$\left(\mathrm{g}_{0}\right) \quad G_{0}:=\lim _{\xi \rightarrow 0^{+}} \frac{\max _{\left(t_{1}, t_{2}\right) \in Q(\xi)} G\left(t_{1}, t_{2}\right)}{\xi^{2}}<+\infty$, and for every $\mu \in\left[0, \mu_{G, \lambda}^{\star}[\right.$, where

$$
\mu_{G, \lambda}^{\star}:=-\frac{1}{8(2 N+3)^{2}\left(\int_{V} g(x) \mathrm{d} \mu\right) G_{0}}\left(1+8 \lambda(2 N+3)^{2}\left(\int_{V} g(x) \mathrm{d} \mu\right) A_{0}\right),
$$

the problem (namely $\left.\left(S_{\lambda, \mu}\right)\right)$ given by

$$
\begin{cases}\Delta u_{1}(x)+a_{1}(x) u_{1}(x)=g(x)\left(\lambda F_{u_{1}}\left(u_{1}(x), u_{2}(x)\right)+\mu G_{u_{1}}\left(u_{1}(x), u_{2}(x)\right)\right), & x \in V \backslash V_{0}, \\ \Delta u_{2}(x)+a_{2}(x) u_{2}(x)=g(x)\left(\lambda F_{u_{2}}\left(u_{1}(x), u_{2}(x)\right)+\mu G_{u_{1}}\left(u_{1}(x), u_{2}(x)\right)\right), & x \in V \backslash V_{0}, \\ \left.u_{1}\right|_{V_{0}}=\left.u_{2}\right|_{V_{0}}=0 & \end{cases}
$$

admits a sequence $\left\{\left(u_{1, n}, u_{2, n}\right)\right\} \subset H_{0}^{1}(V) \times H_{0}^{1}(V)$ of pairwise distinct weak solutions such that $\lim _{n \rightarrow \infty}\left\|\left(u_{1, n}, u_{2, n}\right)\right\|=0$.

We want to conclude with this explicative application.

Example 3.1 Consider the increasing sequence of positive real numbers given by

$$
a_{1}:=2, \quad a_{n+1}:=n ! a_{n}^{2}+2
$$

for every $n \geq 1$. Define the $C^{1}$-function $F: \mathbb{R}^{2} \rightarrow \mathbb{R}$ as follows

$$
F\left(t_{1}, t_{2}\right):= \begin{cases}a_{n+1}^{4} \mathrm{e}^{-\frac{1}{1-\left[\left(t_{1}-a_{n+1}\right)^{2}+\left(t_{2}-a_{n+1}\right)^{2}\right]}+1}, & \text { if }\left(t_{1}, t_{2}\right) \in \bigcup_{n \geq 1} B\left(\left(a_{n+1}, a_{n+1}\right), 1\right), \\ 0, & \text { otherwise, }\end{cases}
$$

where $B\left(\left(a_{n+1}, a_{n+1}\right), 1\right)$ denotes the open unit ball of center $\left(a_{n+1}, a_{n+1}\right)$.

By definition $F$ is non-negative and $F(0,0)=0$. We will denote by $F_{u_{1}}$ and $F_{u_{2}}$ the partial derivative of $F$ with respect to $u_{1}$ and $u_{2}$, respectively. Now, for every $n \in \mathbb{N}$, the restriction $\left.F\left(t_{1}, t_{2}\right)\right|_{B\left(\left(a_{n+1}, a_{n+1}\right), 1\right)}$ attains its maximum at $\left(a_{n+1}, a_{n+1}\right)$, and one has $F\left(a_{n+1}, a_{n+1}\right)=$ $a_{n+1}^{4}$. Clearly

$$
\limsup _{\substack{\left(t_{1}, t_{2}\right) \rightarrow \infty \\\left(t_{1}, t_{2}\right) \in \mathbb{R}_{+}^{2}}} \frac{F\left(t_{1}, t_{2}\right)}{\sum_{i=1}^{2} t_{i}^{2}}=+\infty
$$

owing to

$$
\lim _{n \rightarrow \infty} \frac{F\left(a_{n+1}, a_{n+1}\right)}{2 a_{n+1}^{2}}=+\infty .
$$

On the other hand, by setting $y_{n}=a_{n+1}-1$ for every $n \in \mathbb{N}$, one has

$$
\max _{\left(t_{1}, t_{2}\right) \in Q\left(y_{n}\right)} F\left(t_{1}, t_{2}\right)=a_{n}^{4}, \quad \forall n \in \mathbb{N} .
$$


Then

$$
\lim _{n \rightarrow \infty} \frac{\max _{\left(t_{1}, t_{2}\right) \in Q\left(y_{n}\right)} F\left(t_{1}, t_{2}\right)}{\left(a_{n+1}-1\right)^{2}}=0
$$

and

$$
\liminf _{\xi \rightarrow+\infty} \frac{\max _{\left(t_{1}, t_{2}\right) \in Q(\xi)} F\left(t_{1}, t_{2}\right)}{\xi^{2}}=0 .
$$

The previous computations ensure that all the hypotheses of Theorem 3.1 are satisfied. Then, for every $\lambda \in] 0,+\infty[$, the following problem

$$
\begin{cases}-\Delta u_{1}(x)=\lambda F_{u_{1}}\left(u_{1}(x), u_{2}(x)\right), & x \in V \backslash V_{0}, \\ -\Delta u_{2}(x)=\lambda F_{u_{2}}\left(u_{1}(x), u_{2}(x)\right), & x \in V \backslash V_{0}, \\ \left.u_{1}\right|_{V_{0}}=\left.u_{2}\right|_{V_{0}}=0 & \end{cases}
$$

admits a sequence of (strong) solutions which is unbounded in $H_{0}^{1}(V) \times H_{0}^{1}(V)$.

Remark 3.4 Fix an integer $m \geq 1$. Moreover, let $F: \mathbb{R}^{m} \rightarrow \mathbb{R}$ be a $C^{1}$-function such that $F(0, \cdots, 0)=0$, and $F_{u_{i}}$ denotes the partial derivative of $F$ with respect to $u_{i}$. We explicitly observe that our results hold also for Dirichlet systems of the form

$$
\left\{\begin{array}{l}
\Delta u_{k}(x)+a_{k}(x) u_{k}(x)=\lambda g(x) F_{u_{k}}\left(u_{1}(x), \cdots, u_{m}(x)\right), \quad x \in V \backslash V_{0}, 1 \leq k \leq m, \\
\left.u_{1}\right|_{V_{0}}=\cdots=\left.u_{k}\right|_{V_{0}}=0,
\end{array}\right.
$$

assuming that the functions $a_{k}, g$ satisfy conditions like $\left(\mathrm{h}_{1}\right)$ and $\left(\mathrm{h}_{2}\right)$. For instance, for every $\xi>0$, set

$$
Q_{m}(\xi):=\left\{\left(t_{1}, \cdots, t_{m}\right) \in \mathbb{R}^{m}: \sum_{i=1}^{m}\left|t_{i}\right| \leq \xi\right\}
$$

and

$$
\mathbb{R}_{+}^{m}=\left\{\left(t_{1}, \cdots, t_{m}\right) \in \mathbb{R}^{m}: t_{i} \geq 0, \forall i=1, \cdots, m\right\}
$$

Requiring that

$$
A_{\infty}:=\liminf _{\xi \rightarrow+\infty} \frac{\max _{\left(t_{1}, \cdots, t_{m}\right) \in Q_{m}(\xi)} F\left(t_{1}, \cdots, t_{m}\right)}{\xi^{2}}<+\infty
$$

and

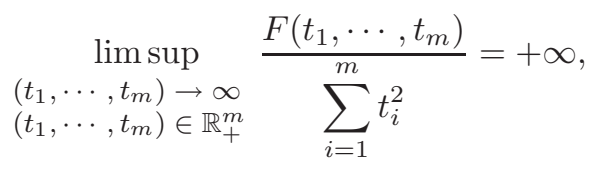

for every

$$
\lambda \in] 0,-\frac{1}{2 m^{2}(2 N+3)^{2}\left(\int_{V} g(x) \mathrm{d} \mu\right) A_{\infty}}[,
$$

there exists an unbounded sequence of weak solutions (in $\prod_{k=1}^{m} H_{0}^{1}(V)$ ) of the above problem. From the above remark one clearly has that Theorem 3.1 extends [15, Theorem 3.2] in the gradient type setting.

Finally, for completeness, among the contributions to the theory of nonlinear elliptic equations on fractals we mention in $[18,24,26,30-31,45]$. The main tools used in these papers to prove the existence of at least one nontrivial solution or multiple solutions for nonlinear elliptic equations with zero Dirichlet boundary conditions are certain minimax results (mountain pass theorems, saddle-point theorems), respectively, and minimization procedures. 


\section{References}

[1] Alexander, S., Some properties of the spectrum of the Sierpiński gasket in a magnetic field, Phys. Rev. B, 29, 1984, 5504-5508.

[2] Barlow, M. T. and Kigami, J., Localized eigenfunctions of the Laplacian on p.c.f. self-similar sets, J. London Math. Soc., 56, 1997, 320-332.

[3] Bartsch, T. and de Figueiredo, D. G., Infinitely many solutions of nonlinear elliptic systems, Progr. Nonlinear Differential Equations Appl., 35, 1999, 51-67.

[4] Bensedik, A. and Bouchekif, M., On certain nonlinear elliptic systems with indefinite terms, Electron. J. Differential Equations, 83, 2002, 1-16.

[5] Boccardo, L. and de Figueiredo, D. G., Some remarks on a system of quasilinear elliptic equations, Nonlinear Differential Equations Appl., 9, 2002, 309-323.

[6] Bockelman, B. and Strichartz, R. S., Partial differential equations on products of Sierpiński gaskets, Indiana Univ. Math. J., 56, 2007, 1361-1375.

[7] Bonanno, G. and D'Aguì, G., On the Neumann problem for elliptic equations involving the $p$-Laplacian, J. Math. Anal. Appl., 358, 2009, 223-228.

[8] Bonanno, G. and D'Aguì, G., Multiplicity results for a perturbed elliptic Neumann problem, Abstr. Appl. Anal., 2010, 2010, 1-10.

[9] Bonanno, G. and Molica Bisci, G., Infinitely many solutions for a boundary value problem with discontinuous nonlinearities, Bound. Value Probl., 2009, 2009, 1-20.

[10] Bonanno, G. and Molica Bisci, G., Infinitely many solutions for a Dirichlet problem involving the $p$ Laplacian, Proc. Roy. Soc. Edinburgh Sect. A, 140, 2010, 737-752.

[11] Bonanno, G., Molica Bisci, G. and O'Regan, D., Infinitely many weak solutions for a class of quasilinear elliptic systems, Math. Comput. Modelling, 52, 2010, 152-160.

[12] Bonanno, G., Molica Bisci, G. and Rădulescu, V., Infinitely many solutions for a class of nonlinear eigenvalue problems in Orlicz-Sobolev spaces, C. R. Acad. Sci. Paris Ser. I, 349, 2011, 263-268.

[13] Bonanno, G., Molica Bisci, G. and Rădulescu, V., Arbitrarily small weak solutions for a nonlinear eigenvalue problem in Orlicz-Sobolev spaces, Monatsh. Math., 165, 2012, 305-318.

[14] Bonanno, G., Molica Bisci, G. and Rădulescu, V., Infinitely many solutions for a class of nonlinear elliptic problems on fractals, C. R. Acad. Sci. Paris Ser. I, 350, 2012, 187-191.

[15] Bonanno, G., Molica Bisci, G. and Rădulescu, V., Variational analysis for a nonlinear elliptic problem on the Sierpiński gasket, ESAIM: Control, Optimisation and Calculus of Variations, 18, 2012, 941-953.

[16] Bozhkova, Y. and Mitidieri, E., Existence of multiple solutions for quasilinear systems via fibering method, J. Differential Equations, 190, 2003, 239-267.

[17] Breckner, B. E., Rădulescu, V. and Varga, C., Infinitely many solutions for the Dirichlet problem on the Sierpiński gasket, Analysis and Applications, 9, 2011, 235-248.

[18] Breckner, B. E., Repovš, D. and Varga, C., On the existence of three solutions for the Dirichlet problem on the Sierpiński gasket, Nonlinear Anal., 73, 2010, 2980-2990.

[19] Ciarlet, P. G., Linear and Nonlinear Functional Analysis with Applications, Society for Industrial and Applied Mathematics (SIAM), Philadelphia, PA, in press.

[20] Clément, Ph., de Figueiredo, D. G. and Mitidieri, E., Positive solutions of semilinear elliptic systems, Comm. Partial Differential Equations, 17, 1992, 923-940.

[21] D'Aguì, G. and Molica Bisci, G., Existence results for an elliptic Dirichlet problem, Matematiche, 1, 2011, 133-141.

[22] D'Aguì, G. and Molica Bisci, G., Infinitely many solutions for perturbed hemivariational inequalities, Bound. Value Probl., 2011, 2011, 1-19.

[23] de Figueiredo, D. G., Semilinear elliptic systems: a survey of superlinear problems, Resenhas IME-USP, 2, 1996, 373-391.

[24] Falconer, K. J., Semilinear PDEs on self-similar fractals, Commun. Math. Phys., 206, 1999, $235-245$.

[25] Falconer, K. J., Fractal Geometry: Mathematical Foundations and Applications, 2nd edition, John Wiley and Sons, Hoboken, NJ, 2003.

[26] Falconer, K. J. and Hu, J., Nonlinear elliptical equations on the Sierpiński gasket, J. Math. Anal. Appl., 240, 1999, 552-573. 
[27] Fukushima, M. and Shima, T., On a spectral analysis for the Sierpiński gasket, Potential Anal., 1, 1992, $1-35$.

[28] Goldstein, S., Random walks and diffusions on fractals, Percolation Theory and Ergodic Theory of Infinite Particle Systems, H. Kesten (ed.), 121-129, IMA Math. Appl., Vol. 8, Springer-Verlag, New York. 1987.

[29] Hai, D. D. and Shivaji, R., An existence result on positive solutions for a class of $p$-Laplacian systems, Nonlinear Anal., 56, 2004, 1007-1010.

[30] $\mathrm{Hu}$, J., Multiple solutions for a class of nonlinear elliptic equations on the Sierpiński gasket, Sci. China Ser. A, 47, 2004, 772-786.

[31] Hua, C. and Zhenya, H., Semilinear elliptic equations on fractal sets, Acta Math. Sci. Ser. B, 29(2), 2009, $232-242$.

[32] Kigami, J., A harmonic calculus on the Sierpiński spaces, Japan J. Appl. Math., 8, 1989, 259-290.

[33] Kigami, J., Analysis on Fractals, Cambridge University Press, Cambridge, 2001.

[34] Kristály, A., Rădulescu, V. and Varga, C., Variational Principles in Mathematical Physics, Geometry, and Economics: Qualitative Analysis of Nonlinear Equations and Unilateral Problems, Cambridge University Press, Cambridge, 2010.

[35] Kusuoka, S., A diffusion process on a fractal, Probabilistic Methods in Mathematical Physics (Katata/Kyoto, 1985, 251-274, Academic Press, Boston, 1987.

[36] Mandelbrot, B. B., How long is the coast of Britain? Statistical self-similarity and fractional dimension, Science, 156, 636-638.

[37] Mandelbrot, B. B., Fractals: Form, Chance and Dimension, W. H. Freeman and Co., San Francisco, 1977.

[38] Mandelbrot, B. B., The Fractal Geometry of Nature, W. H. Freeman and Co., San Francisco, 1982.

[39] Omari, P. and Zanolin, F., An elliptic problem with arbitrarily small positive solutions, Nonlinear Differential Equations, Electron. J. Differential Equations Conf., 5, 2000, 301-308.

[40] Rammal, R., A spectrum of harmonic excitations on fractals, J. Phy. Lett., 45, 1984, 191-206.

[41] Rammal, R. and Toulouse, G., Random walks on fractal structures and percolation clusters, J. Phys. Lett., 44, 1983, L13-L22.

[42] Ricceri, B., A general variational principle and some of its applications, J. Comput. Appl. Math., 113, 2000, 401-410.

[43] Sierpiński, W., Sur une courbe dont tout point est un point de ramification, Comptes Rendus, 160, 1915, 302-305.

[44] Strichartz, R. S., Analysis on fractals, Notices Amer. Math. Soc., 46, 1999, 1199-1208.

[45] Strichartz, R. S., Solvability for differential equations on fractals, J. Anal. Math., 96, 2005, 247-267.

[46] Strichartz, R. S., Differential Equations on Fractals, A. Tutorial (ed.), Princeton University Press, Princeton, 2006.

[47] Zhang, G. Q., Liu, X. P. and Liu, S. Y., Remarks on a class of quasilinear elliptic systems involving the (p,q)-Laplacian, Electron. J. Differential Equations, 2005, 2005, 1-10. 